\title{
Limited Field-of-View Multimodal Sensor Adaptation for Data Association
}

\author{
Sean M. O'Rourke, A. Lee Swindlehurst \\ Center for Pervasive Communications and Computing \\ University of California, Irvine; Irvine, CA 92697 \\ Email: \{orourkes,swindle\}@uci.edu
}

\begin{abstract}
We have investigated the utility of field-of-view adaptation for multimodal sensing in cluttered multi-target environments. Measurement data from multiple integrated sensors are collected at a fusion center, which employs a soft association procedure to integrate them into the estimation procedure. A variance penalty model for the limited fields-of-view property is incorporated into the state estimation procedure. This model also forms the basis of an optimization problem that determines the best next-step sensing parameters for the changing target environment. Numerical simulations demonstrate the benefit of the proposed method for both tracking and association metrics compared to a non-adaptive tracker.
\end{abstract}

\section{INTRODUCTION}

The role of multimodal sensing in multiple target tracking and data fusion research has steadily increased in recent years. By combining multiple sensor types into a single integrated system, the object is to obtain superior performance compared with using the modes individually. A commonly investigated sensor grouping is the combining of active and passive sensor modes, exemplified by the radio-frequency radar/electrooptical imaging (RF/EO) sensor pairing. It has been shown [1] that this pairing results in improved data fusion, reducing misassociation and tracking errors in environments with clutter. These systems have also been shown to benefit from adaptive mode and parameter selection, which forms the basis for the rest of the following discussion.

Adaptive methods for active sensing modes have been extensively studied in a variety of contexts. A notable example is the pioneering work by Kershaw and Evans in [2] on radar waveform adaptation for single target tracking in clutter. Additional examples can be found in the review paper by Sira, et al. [3] and the references within. While a variety of sensing limitations and constraints have been investigated for adaptive sensing, the concept of field-of-view limitations has been mostly limited to the computer vision and camera literature, e.g. [4], [5]. Since a primary purpose of multimodal sensing is to cooperatively compensate for limitations and exploit benefits unique to each mode, it is only natural to consider this effect in the raw adaptive sensing context. In [6], we considered the finite FOV effect in multimodal sensing. We parameterized the FOV effect using penalty functions

This work was supported by the Sensors Directorate of the Air Force Research Laboratory (AFRL/RYRT) under contract number FA8650-08-D1303 to Dynetics, Inc. that depend on the mode, measurement function, and control variables. These penalty functions augment known models of each measurement's noise variance (itself a function of the control) by assigning significantly larger variances to targets outside or nearly outside the FOV.

While [6] considered only the tracking problem assuming perfect target detection and association, in this paper we consider a more realistic case with false alarms and missed detections, and show the advantage of the algorithm in improving data association. The rest of the paper is organized as follows. Section II describes the target-state and sensor measurement models, as well as the data association and state estimation procedures employed in this work. We briefly recapitulate the basic concepts behind the limited FOV penalty models and then describe the proposed optimization scheme, as well as its relation to data association metrics in Section III. Simulation results for both tracking and association based on a challenging example scenario are detailed in Section IV, and Section V provides concluding remarks and commentary.

\section{SySTEM MODELING}

\section{A. Target dynamic models}

We consider a linear model of the target dynamics that operates in discrete time with a sampling period $T$. For simplicity, we only consider two-dimensional movement in the $(x, y)$ plane. At time step $k$, the state vector for the $i$ th target is comprised of position and velocity coordinates $\mathbf{x}_{k}^{i}=\left[\begin{array}{llll}x_{k}^{i} & \dot{x}_{k}^{i} & y_{k}^{i} & \dot{y}_{k}^{i}\end{array}\right]^{T}$, and the state vector is assumed to evolve as

$$
\mathbf{x}_{k+1}^{i}=\mathbf{A}_{k}^{i} \mathbf{x}_{k}^{i}+\mathbf{w}_{k}^{i}
$$

where $\mathbf{A}_{k}^{i}$ is a maneuver-dependent system matrix and $\mathbf{w}_{k}^{i}$ is zero-mean Gaussian process noise with covariance matrix $\mathbf{Q}_{k}^{i}$. We assume further that there are $N_{T, k}$ targets present in the overall surveillance region, and we denote the multitarget state as $\chi_{k}=\left\{\mathbf{x}_{k}^{i}\right\}_{i=1}^{N_{T, k}}$. The number of targets $N_{T, k}$ is assumed to be constant and known a priori.

\section{B. Multimodal sensing models}

Suppose we observe the aforementioned target environment with $N_{S}$ homogenous multimodal sensors distributed in the surveillance region, with each sensor $p$ comprising of $M$ modes. Each mode $s$ produces a number of raw returns 
that are preprocessed in a manner that produces kinematic measurements based on the target state vectors and clutter, with target detection probability $P_{D,(p, s)}$ and false alarm rate $P_{F A,(p, s)}$. Furthermore, we can parameterize the $(p, s)$ th sensor-mode's operation at time $k$ by the vector $\mathbf{p}_{(p, s), k}$. In the interests of notational simplicity, the paired sensor-mode indexing $(p, s)$ is collapsed into a single (pseudo-)sensor index $j$ defined by the mapping $j=M(p-1)+s$. At time $k$, $m_{j, k}$ detected and validated measurement vectors are obtained from sensor $j$, denoted by the set $\mathcal{Y}_{j, k}=\left\{\mathbf{y}_{l_{j}, j, k}\right\}_{l_{j}=1}^{m_{j, k}}$. If the $l_{j}$ th measurement originated from the $i$ th target, then $\mathbf{y}_{l_{j}, j, k}$ is given by:

$$
\mathbf{y}_{l_{j}, j, k}^{i}=\mathbf{c}_{j}\left(\mathbf{x}_{k}^{i}\right)+\mathbf{v}_{j, k}^{i}
$$

where $\mathbf{c}_{j}(\cdot)$ is the (possibly non-linear) mode measurement function and $\mathbf{v}_{j, k}^{i}$ is a zero-mean Gaussian random vector denoting measurement noise with system parameter-dependent covariance $\mathbf{R}_{j}\left(\mathbf{p}_{j, k}\right)$. We assume these noise sequences are uncorrelated with both the target process noise and the measurement noise from any other sensors or targets. Measurements that are not target-originated are clearly false alarms/clutter, which are assumed to be uniformly distributed throughout the $j$ th sensor's validation region that has a volume $V_{j, k}$ at time $k$ - that is, the probability distribution function for a clutter measurement $\overline{\mathbf{v}}_{j, k}$ is $p\left(\overline{\mathbf{v}}_{j, k}\right)=1 / V_{j, k}$. The number of false alarms within a given validation region is a random variable modeled by a prior that depends on the available information about the clutter environment. In our case, we assume the persensor clutter density $\bar{\lambda}_{j}$ is known, hence the number of false alarms in the volume is Poisson distributed with parameter $\bar{\lambda}_{j} V_{j, k}$.

With the general model in place, we describe the specific models used for the specific RF/EO mode pairing. Assume each sensor $j$ is located at the coordinates $\left(\bar{x}_{j}, \bar{y}_{j}\right)$. The RF mode forms its kinematic measurements from matched filter-processed returns in range-Doppler space, resulting in range and range-rate measurements. In particular, the measurement function from the $i$ th target is $\mathbf{c}_{j, R F}^{i}=\left[\begin{array}{ll}r_{j}^{i} & \dot{r}_{j}^{i}\end{array}\right]^{T}$, with components given by $r_{j}^{i}=\sqrt{\left(x^{i}-\bar{x}_{j}\right)^{2}+\left(y^{i}-\bar{y}_{j}\right)^{2}}$ and $\dot{r}_{j}^{i}=\left(\dot{x}^{i}\left(x^{i}-\bar{x}_{j}\right)-\dot{y}^{i}\left(y^{i}-\bar{y}_{j}\right)\right) / r_{j}^{i}$. Assuming that the RF mode is parameterized by a per-pulse bandwidth $B_{j}$ and a pulse repetition frequency $P R F_{j}$, the measurement covariance matrix is given by $\mathbf{R}_{j, R F}=$ $(1 / 12) \operatorname{diag}\left\{\left(c / 2 B_{j}\right)^{2},\left(\lambda P R F_{j} / 2 N_{f f t}\right)^{2}\right\}$, where $\lambda$ is the carrier wavelength, $N_{f f t}$ is the Doppler integration length (FFT size), and $\operatorname{diag}\{\cdot\}$ creates a diagonal matrix from its entries. The EO mode measures the target azimuth: $\mathbf{c}_{j, E O}^{i}=\eta_{j}^{i}$, where $\eta_{j}^{i}=\arcsin \left(\left(x^{i}-\bar{x}_{j}\right) / r_{j}^{i}\right)$. If we assume the mode captures a one-dimensional image $N_{p i x}$ pixels wide with a detector/pixel angular subtense of $\alpha$, the noise variance is $\mathbf{R}_{j, E O}=\alpha^{2} / 12$ [7]. For each of these measurement variances, we have assumed that the measurement noise is uniformly distributed in the given resolution cell/pixel and that only one target return originates from it.

\section{Estimation and Association methods}

Tracking algorithms require a data association method to deal with false alarms and missed detections to determine measurement origins and incorporate them into a state estimation procedure. In this paper, we will consider the multisensor joint probabilistic data association (MSJPDA) method first detailed in [8], but our characterization of the problem is compatible with other common methods [9]. More specifically, we implement the sequential MSJPDA [10], which uses multiple single-sensor JPDAs to sequentially process each sensor's measurements, initializing the next iteration with the filtered outputs of the previous step.

Assuming an extended Kalman filter (EKF) is employed, the prediction step is identical to the standard EKF, resulting in an estimate of the dynamic state $\hat{\mathbf{x}}_{k \mid k-1}^{i}$ and error covariance $\mathbf{P}_{k \mid k-1}^{i}$ for each target $i$ at time $k$. Treating the various modes of each sensor as independent sensors results in $N_{\bar{S}}=N_{S} M$ total sensor-modes. If we define the intermediate filtered estimate and covariance for the $i$ th target from the $j$ th sensor/mode as $\hat{\mathbf{x}}_{F, j}^{i}$ and $\mathbf{P}_{F, j}^{i}$ respectively (where the subscript $F$ denotes "filtered"), with initial values $\hat{\mathbf{x}}_{F, 0}^{i}=\hat{\mathbf{x}}_{k \mid k-1}^{i}$, then $\mathbf{P}_{F, j}^{i}=\mathbf{P}_{k \mid k-1}^{i}$. Each update iteration starts by calculating the joint probabilities $\beta_{l_{j}, j}^{i}$ of the $l_{j}$ th measurement being a true measurement of the $i$ th target (including the no-measurement probability $\beta_{0, j}^{i}$ ) as in the JPDA [11]. Then, we generate the intermediate updated per-target state estimate and covariance with the recursions

$$
\begin{aligned}
\hat{\mathbf{x}}_{F, j}^{i} & =\hat{\mathbf{x}}_{F, j-1}^{i}+\mathbf{K}_{j}^{i} \overline{\boldsymbol{\nu}}_{j}^{i} \\
\mathbf{P}_{F, j}^{i} & =\mathbf{P}_{F, j}^{i}-\left[1-\beta_{0, j}^{i}\right] \mathbf{K}_{j}^{i} \mathbf{S}_{j}^{i} \mathbf{K}_{j}^{i T} \\
& +\mathbf{K}_{j}^{i}\left[\sum_{l_{j}=1}^{m_{j, k}} \beta_{l_{j}, j}^{i} \boldsymbol{\nu}_{l_{j}, j}^{i} \boldsymbol{\nu}_{l_{j}, j}^{i T}-\overline{\boldsymbol{\nu}}_{j}^{i} \overline{\boldsymbol{\nu}}_{j}^{i T}\right] \mathbf{K}_{j}^{i T}
\end{aligned}
$$

where we define the per-measurement innovation $\boldsymbol{\nu}_{l_{j}, j}^{i}=$ $\mathbf{y}_{l_{j}, j}-\mathbf{c}_{j}\left(\mathbf{x}^{i}\right)$ and the combined innovation $\overline{\boldsymbol{\nu}}_{j}^{i}=$ $\sum_{l_{j}=1}^{m_{j, k}} \beta_{l_{j}, j}^{i} \boldsymbol{\nu}_{l_{j}, j}^{i}$. The filter gain $\mathbf{K}_{j}^{i}$ depends on the underlying estimation procedure, as does the innovation covariance $\mathbf{S}_{j}^{i}$. Under the EKF assumption, $\mathbf{K}_{j}^{i}=\left(\mathbf{P}_{F, j-1}^{i}\right)^{-1} \mathbf{C}_{j}^{i}\left(\mathbf{S}_{j}^{i}\right)^{-1}$ and $\mathbf{S}_{j}^{i}=\mathbf{C}_{j}^{i} \mathbf{P}_{F, j-1}^{i} \mathbf{C}_{j}^{i T}+\mathbf{R}_{j}^{i}$, where $\mathbf{C}_{j}^{i}$ is the Jacobian of the measurement function evaluated at $\hat{\mathbf{x}}_{F, j-1}^{i}$. The final updates are given by the filtered values from the last sensor; that is, $\hat{\mathbf{x}}_{k \mid k}^{i}=\hat{\mathbf{x}}_{F, N_{\bar{S}}}$ and $\mathbf{P}_{k \mid k}^{i}=\mathbf{P}_{F, N_{\bar{S}}}^{i}$.

\section{AlgORITHM DESCRIPTION}

\section{A. Finite Field-of-View Modeling with Penalty Functions}

As mentioned above, for a measurement to be a true return, the target must not only be detected, but also visible to the sensor in the first place. This requires targets to remain within a sensor mode's field-of-view (FOV), which may not always be the case. In order to account for this effect, we previously introduced in [6] the idea of augmenting each mode's measurement covariance with penalty functions dependent on the mode measurement function, the FOV, and the sensing parameters. These functions effectively approximate the large 
uncertainty associated with a true target that lies outside a sensor mode's FOV. Mathematically, for a one dimensional measurement with uncorrected variance $\sigma_{m i n}^{2}$, inclusion of the finite field-of-view effect results in the augmented variance $\sigma_{L F O V}^{2}=\sigma_{\text {min }}^{2}(\mathbf{p})+\phi(\mathbf{c}(\mathbf{x}), F O V(\mathbf{p}))$ where $\phi(\cdot)$ is the penalty function; $\mathbf{c}(\cdot)$ \& $F O V(\cdot)$ are sensor (mode) dependent state measurement and finite FOV functions, respectively, and $\mathbf{p}$ is a vector of mode-specific control parameters. The penalty approximates an indicator function, and there are many possible choices for $\phi(\cdot)$, e.g. a hyperbolic tangent approximation to the step function, the log-barrier function, etc., which ultimately affect the convergence of the numerical optimization and the nature of the resulting policies.

For RF/EO mode pairing, the FOV bounds are easily obtainable. For the RF mode, given the speed of light $c$, the maximum unambiguous range and range-rate define the combined fields-of-view as $F O V(r)=[0, \lambda \mathrm{PRF} / 2]$ meters and $F O V(\dot{r})=[0, c / 2 \mathrm{PRF}]$ meters/sec where $\lambda, B$ and $P R F$ are as defined above. For the EO mode characterized as above, the field-of-view for the received azimuth measurement is defined by the image size and the angle subtended by each pixel, or $|F O V(\eta)| \leq \frac{N_{p i x} \alpha}{2}$ radians.

This formulation augments the MSJPDA/EKF scheme from Section II-C by replacing the solely parameter-dependent sensor/mode covariance $\mathbf{R}_{j}$ with the parameter and target state dependent $\tilde{\mathbf{R}}_{j}^{i}=\mathbf{R}_{j}+\Phi_{j}^{i}$, where $\Phi_{j}^{i}$ is a matrix formed from the per-mode penalties above. However, since $\tilde{\mathbf{R}}_{j}^{i}$ requires evaluating the penalty at the actual target state (which is unknown), we instead use the estimates of each target state from each stage of the sequential MSJPDA, and thus introduce an estimated penalized covariance $\hat{\tilde{\mathbf{R}}}_{j}^{i}$.

\section{B. Parameter Optimization Scheme \& Modifications in Clutter}

In order to mitigate the field-of-view effect, we adaptively select the next-step set of sensing parameters $\mathbf{p}_{k+1}$ (and thus, indirectly, each mode's FOV) so as to improve some tracking or association performance metric. As before, tracking metrics like mean-square state estimation error and next-step information increase [12] remain important, since accurately determining target position is a primary purpose of these types of data fusion systems. However, in uncertain measurement environments, it is also critical to examine costs that include association metrics. An example objective might be to maximize the overall probability of correct association for a particular (sub)set of sensing modes. For soft association methods like the one presented above in Section II-C, however, analytically formulating this metric is difficult due to the non-deterministic nature of measurement-to-track hypothesis generation. A hybrid approach to managing the tracking and association goals is to optimize a function of the validation regions for each mode, as advanced in [2], which affects both association probabilities and tracking error. Our approach [6] attempts to only minimize tracking error, but as we will show in Section IV, this cost function also results in benefits for association as well. Our primary cost function is derived from the covariance update form of the standard EKF; ultimately, we maximize the next-step updated information subject to a worst-case-target minimum threshhold. Incorporating the constraints, the final optimization problem is as in [6]:

$$
\begin{aligned}
\max _{\mathbf{p}_{k+1}} & \operatorname{tr}\left(\mathcal{P}_{k+1 \mid k} \mathcal{C}_{k+1}^{T} \hat{\tilde{\mathcal{R}}}^{-1}\left(\mathbf{p}_{k+1}\right) \mathcal{C}_{k}\right) \\
\text { s.t. } & \min _{i} \operatorname{tr}\left(\mathcal{P}_{k+1 \mid k}^{(i)} \mathcal{C}_{k+1}^{(i) T} \hat{\tilde{\mathcal{R}}}^{(i)-1}\left(\mathbf{p}_{k+1}\right) \mathcal{C}_{k+1}^{(i)}\right) \geq t \\
& \mathbf{l} \preceq \mathbf{p}_{k+1} \preceq \mathbf{u}
\end{aligned}
$$

where $\preceq$ indicates elementwise inequality, $t$ is the minimum information threshold, and $\mathbf{l}, \mathbf{u}$ are system-imposed lower \& upper bounds on the parameter vector. The block matrices $\mathcal{P}_{k+1 \mid k}, \mathcal{C}_{k+1}^{T}$ and $\hat{\tilde{\mathcal{R}}}^{-1}\left(\mathbf{p}_{k+1}\right)$ are formed by concatenating first by sensor, then by target, the per-target/per-sensor error covariances $\mathbf{P}_{j}^{i}$, measurement Jacobian matrices $\mathbf{C}_{j}^{i}$, and augmented measurement covariances $\hat{\tilde{\mathbf{R}}}_{j}^{i}$ (evaluated at the nextstep predicted state $\hat{\mathbf{x}}_{k+1 \mid k}^{i}$ ), respectively. We note that it is possible to augment our cost function with the hybrid covariance approximations or modified Riccati equations presented in [10], but these result in significantly more complex cost functions that require further investigation.

\section{Simulation And Results}

The following example demonstrates the beneficial behavior of our model in a cluttered sensing environment. Consider the "nearly crossing" target scenario shown in Fig. 1. Over 500 seconds, two targets approach each other from the west and south, respectively, executing sharp 90 degree turns to maneuver away before interception. This scenario contains significant elements that challenge the adaptive method: potentially unresolvable target states, depending on the measurement accuracy; and a "stretched" overall area of interest throughout the tracking period, due to the targets splitting off and undergoing multiple maneuvers. Assume that we observe the targets using two paired RF/EO sensor systems, located as in Fig 1, each initialized with identical sensing parameters and fields-of-view. All modes operated with a probability of detection $P_{D}=0.9 \&$ assumed clutter density $\bar{\lambda}=10^{-5}$. Each sensor's measurements are synchronized, made at a defined sampling rate of $T=1$ second, and arrive at a central fusion center for association and inclusion into the state estimation procedure, which is initialized with target positions randomly distributed around the actual positions. We obtained our results from a set of 200 Monte Carlo simulations.

The first result, shown in Fig. 2, echoes the results previously observed in non-cluttered sensing environments. The adaptive method results in generally lower tracking error than the comparable non-adaptive method for both targets. The most substantial difference occurs after the turn maneuvers (around 300 seconds), where the non-adaptive method diverges significantly, but the adaptive method gracefully handles the model mismatch and is able to recover. Similar results have been observed for varying levels of clutter density and probability of detection, though as these parameters increase (decrease), both methods suffer from the degraded sensing environment. 


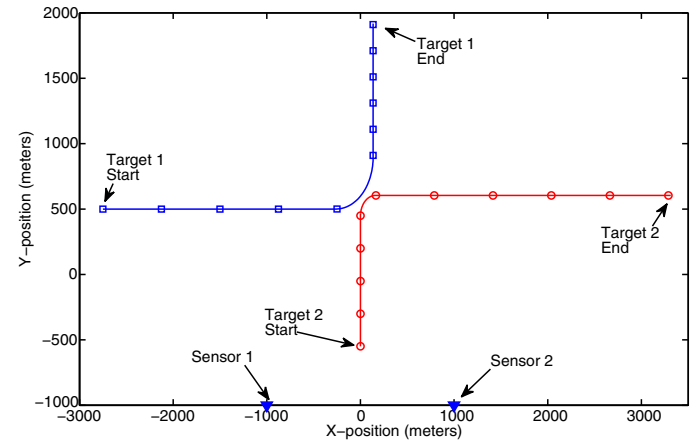

Fig. 1. Example target scenario (shapes mark position every $50 \mathrm{sec}$.)

Using the results of the same Monte Carlo trials as above, we determined how often each method correctly identified the source of each measurement for each sensor and sensing mode. Since the MSJPDA is a soft method, the hypotheses with the largest association probabilities were chosen as the "true" values. Fig. 3 shows the probability of correct association given that at least one measurement was made. Again, the adaptive method outperforms the non-adaptive method, showing better correct association probability across all modes and sensors. This is particularly true for the EO sensing mode, where the probability generally doubles for both sensors. It is interesting to note that the adaptive method frequently assigned the EO parameters to correspond to small FOVs/high accuracy, resulting in many periods of no measurement, more than the non-adaptive method. However, this implies that the adaptive method takes into account the potential for clutteronly measurements and actively avoids it by reducing the potential area for clutter to its smallest allowable value.
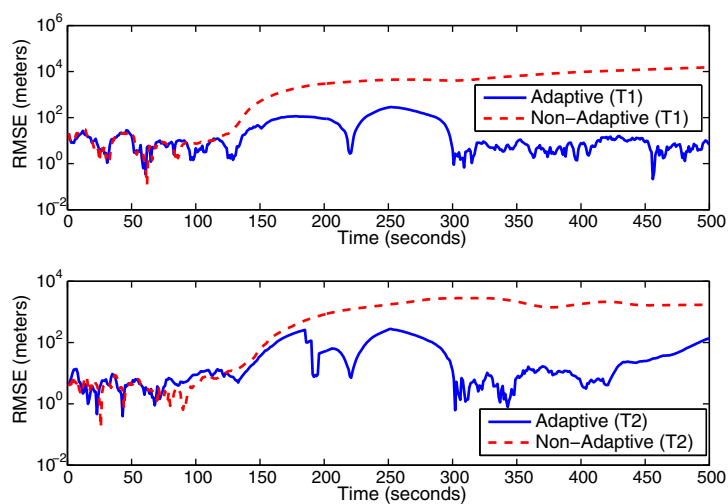

Fig. 2. Position error comparison for the given scenario

\section{CONCLUSiOnS}

We analyzed the behavior of the limited FOV penalty formulation and adaptation algorithm in cluttered sensing environments. We demonstrated how the previously-considered penalty functions and optimization scheme can be incorporated

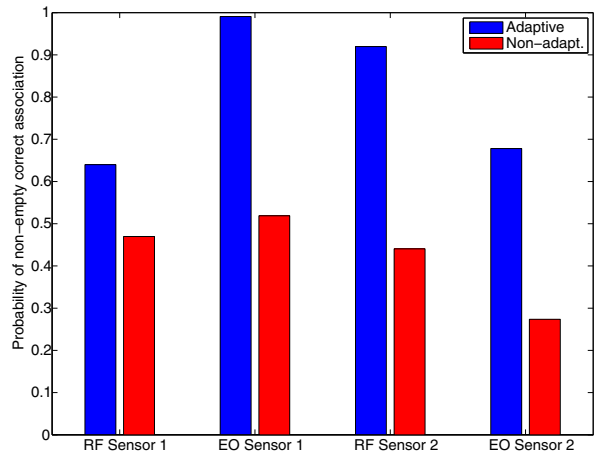

Fig. 3. Comparison of correct association probabilities

into a typical data association scheme. Additionally, methods of augmenting this optimization scheme were discussed and justified. Using numerical simulations, we demonstrated that adaptive FOV selection has multiple benefits in clutter. First, the adaptive method exhibits superior tracking performance, especially when a model mismatch occurs. Second, this tracking performance is accompanied by improved data association, partially as an effect of more selective FOV choices.

\section{REFERENCES}

[1] E. Blasch and C. Yang, "Ten methods to fuse GMTI and HRRR measurements for joint tracking and identification," in Proceedings of the 7th International Conference on Information Fusion (FUSION'04), 2004, pp. 1006-1013.

[2] D. Kershaw and R. Evans, "Waveform selective probabilistic data association," IEEE Transactions on Aerospace and Electronic Systems, vol. 33, no. 4, pp. 1180-1188, Oct. 1997.

[3] S. P. Sira, Y. Li, A. Papandreou-Suppappola, D. Morrell, D. Cochran, and M. Rangaswamy, "Waveform-agile sensing for tracking," IEEE Signal Processing Magazine, vol. 26, no. 1, pp. 53-64, Jan. 2009.

[4] A. Mittal and L. S. Davis, "A General Method for Sensor Planning in Multi-Sensor Systems: Extension to Random Occlusion," International Journal of Computer Vision, vol. 76, no. 1, pp. 31-52, Jul. 2007.

[5] J. Zhao, S.-C. S. Cheung, and T. Ngyuen, "Optimal Visual Sensor Network Configuration," in Multi-Camera Networks: Principles and Applications, H. K. Aghajan and A. Cavallaro, Eds. Oxford: Academic Press, 2009, ch. 6, pp. 139-162.

[6] S. M. O'Rourke and A. L. Swindlehurst, "Closed-loop tracking using multimodal RF/EO sensors," in 2010 Conference Record of the Forty Fourth Asilomar Conference on Signals, Systems and Computers. IEEE, Nov. 2010, pp. 1662-1666.

[7] J. Lloyd, "Fundamentals of Electro-Optical Imaging Systems Analysis," in The Infrared \& Electro-Optical Systems Handbook v. 4, M. C. Dudzik, Ed. Ann Arbor, Michigan and Bellingham, Washington: Infrared Information Analysis Center and SPIE Optical Engineering Press, 1993, ch. 1, pp. 1-54.

[8] L. Pao, "Centralized multisensor fusion algorithms for tracking applications," Control Engineering Practice, vol. 2, no. 5, pp. 875-887, Oct. 1994.

[9] S. Blackman and R. Popoli, Design and Analysis of Modern Tracking Systems. Norwood, MA: Artech House, 1999.

[10] C. W. Frei and L. Y. Pao, "Alternatives to Monte-Carlo simulation evaluations of two multisensor fusion algorithms," Automatica, vol. 34, no. 1, pp. 103-110, Jan. 1998.

[11] Y. Bar-Shalom and X.-R. Li, Multitarget-Multisensor Tracking: Principles and Techniques. YBS Publishing, 1995.

[12] P. Zhan, D. Casbeer, and A. L. Swindlehurst, "Adaptive Mobile Sensor Positioning for Multi-Static Target Tracking," IEEE Transactions on Aerospace and Electronic Systems, vol. 46, no. 1, pp. 120-132, Jan. 2010. 